\title{
Real Time Access to Multiple GPS Tracks
}

\author{
Karol Waga, Andrei Tabarcea, Radu Mariescu-Istodor and Pasi Fränti \\ Speech and Image Processing Unit, School of Computing, University of Eastern Finland, Joensuu, Finland \\ $\{k$ waga, tabarcea, radum, franti\}@cs.uef.fi
}

Keywords: Geographic Information Systems, GPS Tracks, Trajectory Storage and Visualization.

\begin{abstract}
Increasing availability of mobile devices with GPS receiver gives users the possibility to record and share a variety of location-based data, including GPS tracks. We describe a complete real-time system for acquisition, storage, querying, retrieval and visualization of GPS tracks. The main problems faced are how to store the data, how to access and how to visualize large amount of data. We propose to reduce the quantity of the data to be visualized, without affecting visualization quality. In order to achieve this, our system uses a fast polygonal approximation algorithm for different map scales along with a bounding box solution.
\end{abstract}

\section{INTRODUCTION}

Mobile devices with geo-positioning facilitate the acquisition of location-based data. This allows people to track their outdoor movements while performing physical exercises or when traveling. Companies can manage their geographical information in real-time (Martín et al., 2008) and track the movement of their own vehicles in order to solve problems such as fleet management (Jakobs et al., 2001) or traffic congestion (McCullough et al., 2011). The collected tracks are usually uploaded to an online system in order to be viewed, managed and analyzed. However, accessing and visualizing large amount of data is time consuming.

We present MOPSI Routes, a complete system for storage, retrieval and visualization of GPS tracks that overcomes the most common disadvantages of similar systems. For example, existing real-time web based systems, such as www.gmapgis.com and www.gpsvisualizer.com, do not have the possibility to plot large number of points and tracks on the map. In such cases, displaying becomes slow and visualizing overlapping tracks is difficult. Other solutions, such as TopoFusion (Morris et al., 2004), propose combining and intersecting GPS tracks in order to create trails and minimize the data needed to be displayed, although the goal, creating a GPS network of trails, is different. Our solution is to display all the recorded tracks in real time by reducing the number of points that are plotted. This is done by fast multi-resolution polygonal approximation algorithm described in (Chen et al., 2012), which achieves better approximation result than the existing competitive methods. Furthermore, we minimize the time needed for drawing by using a bounding box solution for plotting only the points that are visible to the user.

MOPSI Routes is available as a part of MOPSI services (cs.uef.fi/mopsi) and addresses the issues of storage, querying, retrieval and visualization of GPS tracks, first described in (Waga et al., 2012b). Users voluntarily upload their GPS tracks using our mobile application, which is available for most modern mobile operating systems (Android, Windows Phone, iOS and Symbian).

Similar research projects include GeoLife (Zheng et al., 2008), the system presented in (Alahakone et al., 2009) and StarTrack (Ananthanarayanan et al., 2009).

GeoLife (Zheng et al., 2008) is a project which focuses on visualization, organization, fast retrieval and understanding of GPS track logs. The main goal of the project is understanding people lives based on raw GPS data. The main contribution is visualizing GPS data over digital maps by indexing the GPS trajectories based on uploading behavior of users. Similarly to MOPSI Routes, tracks are searched using spatial range and time query.

The tool described in (Alahakone et al., 2009) is used for manipulating, integrating and displaying geographical referenced information. The main purposes for the tool are path planning and navigation of mobile objects. The tool can be used in 


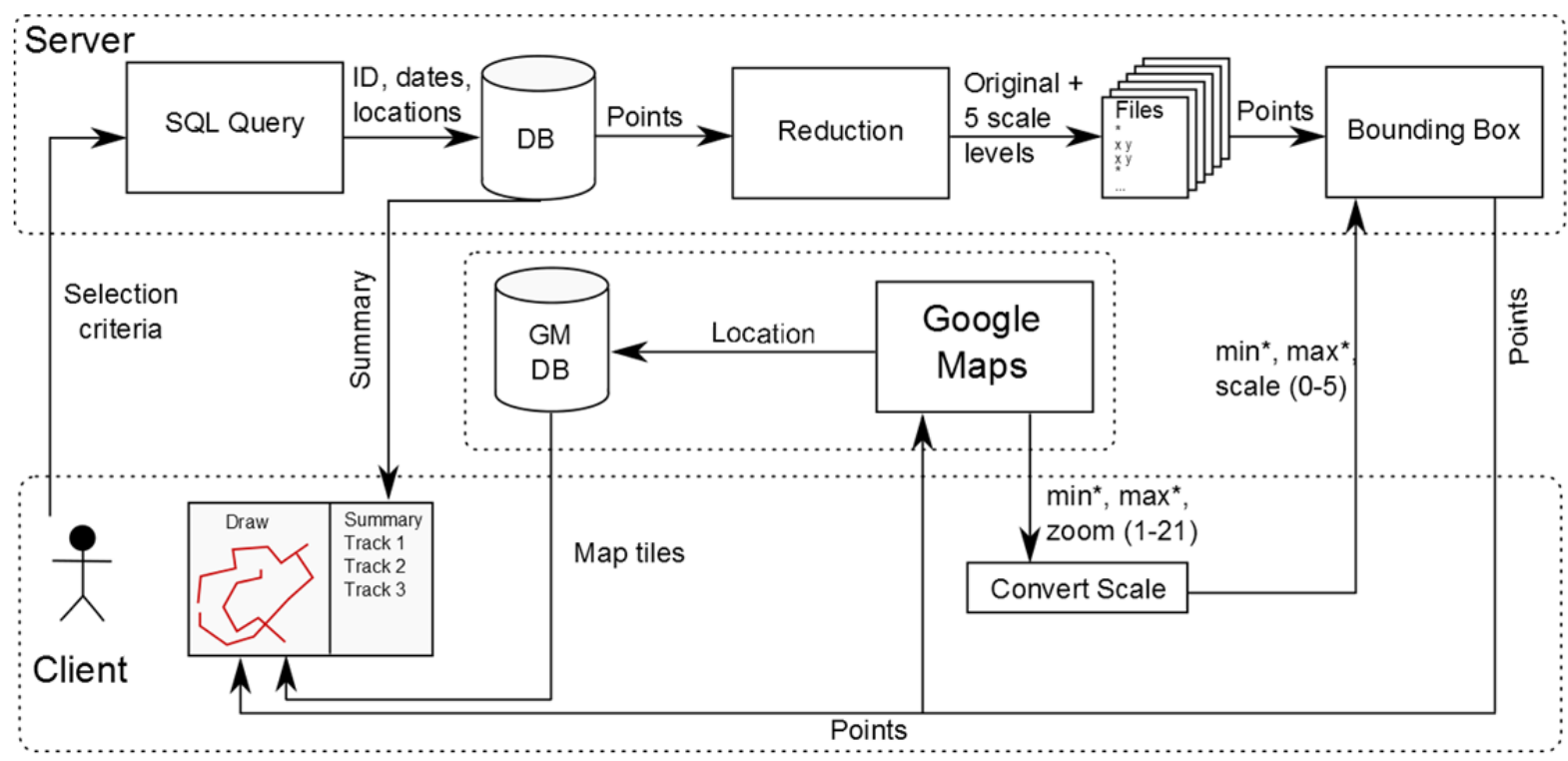

Figure 1: Typical Workflow of MOPSI Routes.

several applications such as: tracking, fleet management, security management and industrial robot navigation. Similarly to our system, a spatial database is used for storing tracks and points and Google Maps API to display the tracks. It presents a general approach in handling GPS data and it can be used in a variety of applications that use track recording, navigation and track planning. It requires that the user selects the points and defines the tracks, whereas our application automatically detects and segments the tracks.

StarTrack (Ananthanarayanan et al., 2009) and its improved version (Haridasan et al., 2010) describe tracks of coordinates as high-level abstraction for various types of location-based applications. The system supports recording, comparison, clustering and querying tracks. Experimental results show that the system is efficient and scalable up to 10.000 tracks. The improved version was extended to operate on collections of tracks, delay query executions and permit caching of query results. Other improvements are canonicalization based on road networks, and use of track trees for similarity.

\section{SYSTEM DESCRIPTION}

MOPSI Routes can be accessed at cs.uef.fi/mopsi/routes. The typical workflow of the system is presented in Fig. 1, whereas Fig. 2 shows example of tracks collection from one user.

In the first step, user selects the tracks to be displayed by several criteria such as time, location, duration and length. Tracks that match the criteria are retrieved from database and processed before displaying to the user. During the processing phase, the points belonging to the retrieved tracks undergo approximation process that reduces the number of points needed for the specific map scales. Points that are outside the visible area of the map are omitted by applying a bounding box. In the final step, the remaining points are shown on the map and the user can browse through them using map view (panning and zooming) or using list view to see additional information and statistics of each route.

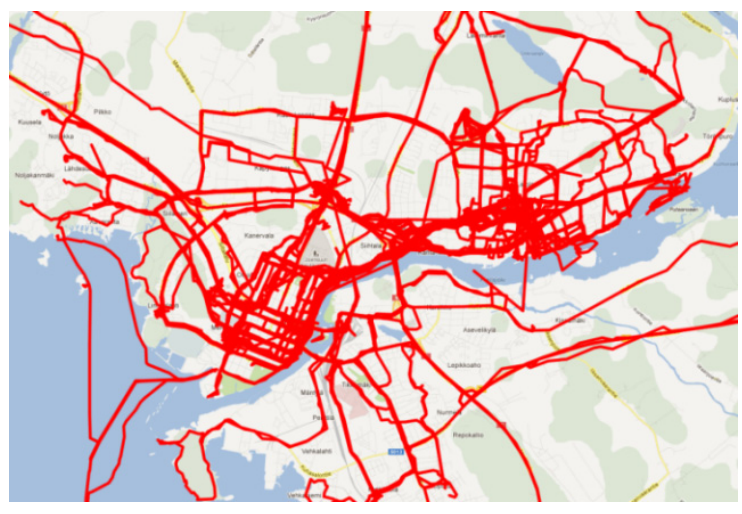

Figure 2: Example of user tracks collection.

\subsection{Data Acquisition and Storage}

MOPSI allows collecting tracking data using smartphones. The mobile application records the user's location and timestamp at a predefined 
interval (usually 1-4 seconds). The data is saved to database on server immediately if internet connection is available, or buffered on the device if internet connection is not available or the application is in offline mode.

Tracks are first saved as individual points in the database, and track objects are created and updated real time when new points are received. Each track object contains not only the points but includes several basic statistics such as start and end time, bounding box and number of points. Segmentation and classification statistics are also stored. Analysis and classification of GPS tracks is described in details in (Waga et al., 2012a). Furthermore, each track is stored in its original and in a simplified form with reduced number of points. The approximated tracks are computed for 5 different zoom levels in order to speed up the visualization process. This limits the number of points drawn on the map without losing significant information about the shape of the GPS track. The analyzed and the approximated tracks are computed immediately when the points are uploaded.

The tracks are created and updated real time and tracking points are handled immediately after they have been uploaded. This process requires maintaining and updating track statistics and information constantly when user is recording a new track. To ensure this, there is a process running constantly on server that checks periodically (every 1 minute) if any track needs to be updated. When new tracking points are uploaded, they are either used for creating a new track object or merged with the existing points and inserted into list of the track's points in time order. The existing tracks are updated in the case that new tracking points belonging to an older track are received with significant delay caused, for example, by poor internet connection.

\subsection{Different Map Scales}

The tracks recorded in our system carry far more data than needed for visualization. Full data is needed for analysis, and therefore, complete GPS tracks must be stored. However, in the rendering process for a web browser, reduced number of points is sufficient to present the shape of track to user. We apply here a multi-resolution polygonal approximation algorithm described in (Chen et al., 2012). The algorithm is fast and achieves good quality approximation of the tracks. It is applied to every track and returns approximation of a track in 5 different map scales. The algorithm time complexity is $\mathrm{O}(\mathrm{N})$ (Chen et al., 2012) and the results are stored to avoid running algorithm repeatedly when the same track is displayed again.

Figure 3 shows an example of the original and approximated tracks. The original track contains 575 points and it is approximated in different map scales with 44, 13, and 6 points respectively. Suitable approximation error tolerance is selected for each map scale, and the visualization quality is not affected by the approximation, but rendering time is reduced significantly.

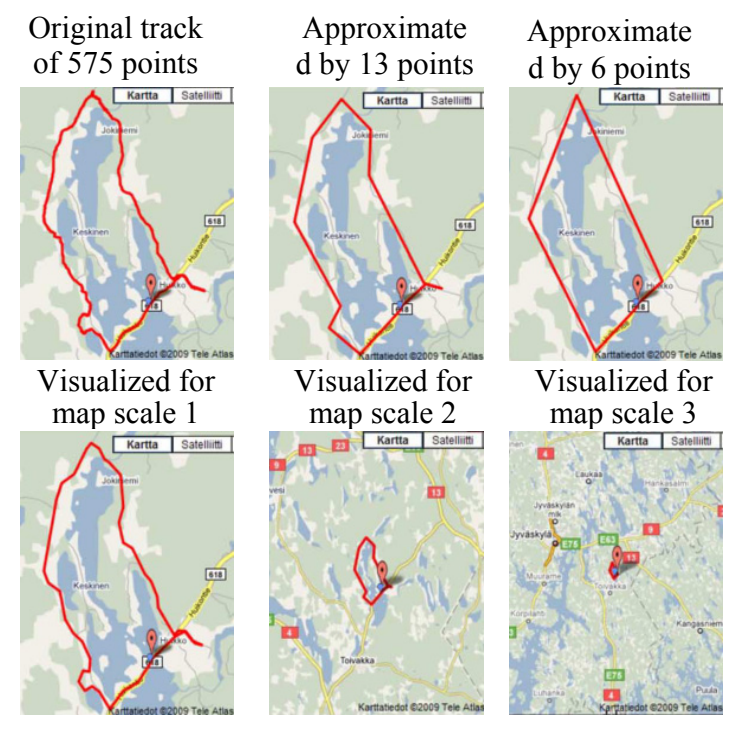

Figure 3: Visualization of a sample track.

\subsection{Bounding Box}

The purpose of the bounding box is to draw on the map only the points that are visible to user, see Fig. 4. Therefore, we select only points that user will see using the current map scale and location (bounding box of the map) at the moment of query. In addition, we draw also points that are outside the bounding box, but within immediate neighborhood $(50 \%$ extension of screen size). In this way, we allow fast panning and zooming.

The bounding box is implemented as a function that gets coordinate of north, east, south and west of the map visible on the screen. Map scale is also passed, so that points from the correct approximation can be selected. The function is applied to every track and for every point it checks if the point lies inside the bounding box. Time complexity of the bounding box is linear and it is computed entirely on server. 


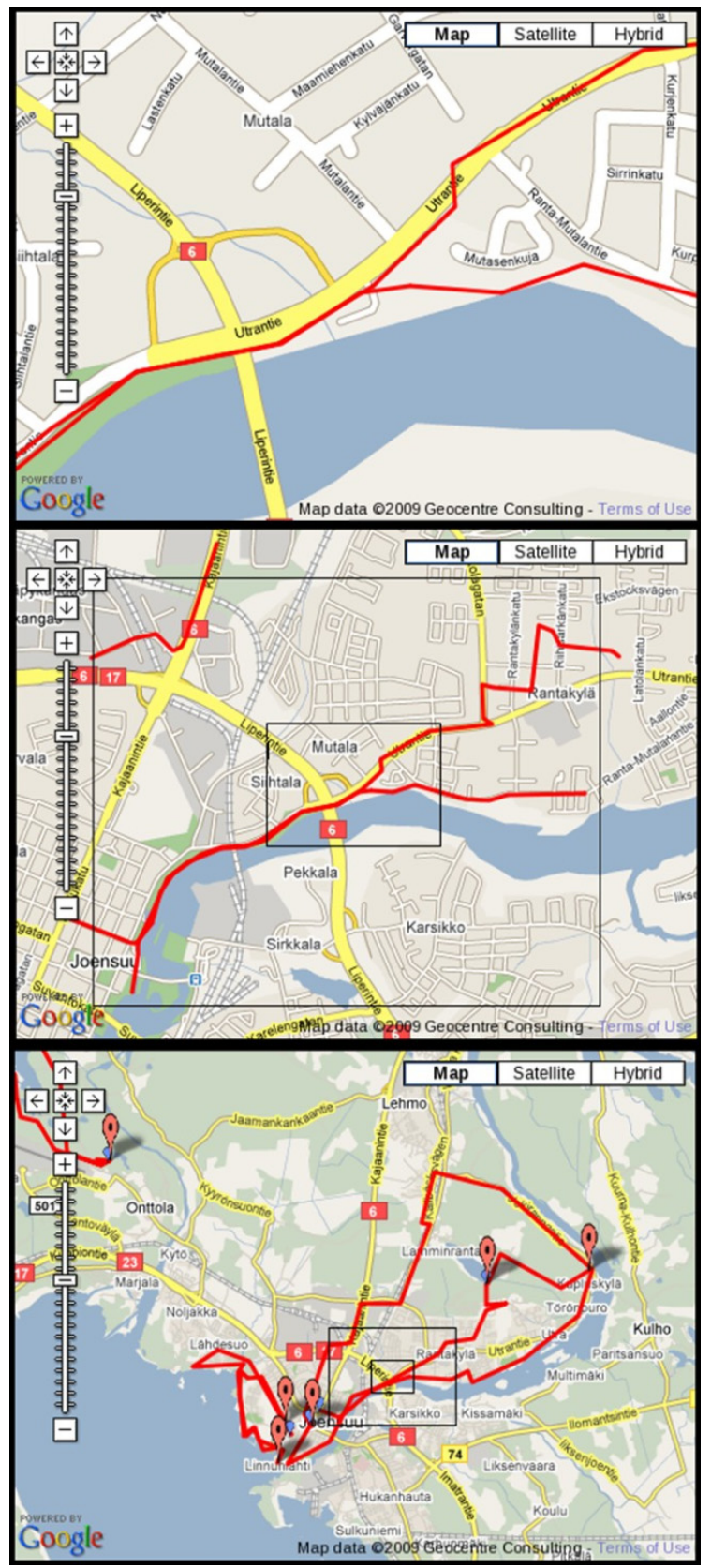

Figure 4: How the bounding box works (from top to bottom): what user sees on screen, what is drawn on map, all tracks selected.

\subsection{Displaying Tracks on Map}

In MOPSI, we use Google Maps to visualize the data (see Fig. 5). However, user can select different type of maps that are displayed as overlay over Google Maps. We support OpenStreetMaps and detailed orienteering maps in Joensuu area where most MOPSI users come from.
There are several search options available. The main search criterion is time, thus only tracks in the selected time period are shown. In addition, other criteria can be applied. For example, tracks can be filtered by minimum and maximum length and duration. Moreover, it is possible to search for tracks that start and end around a certain location.

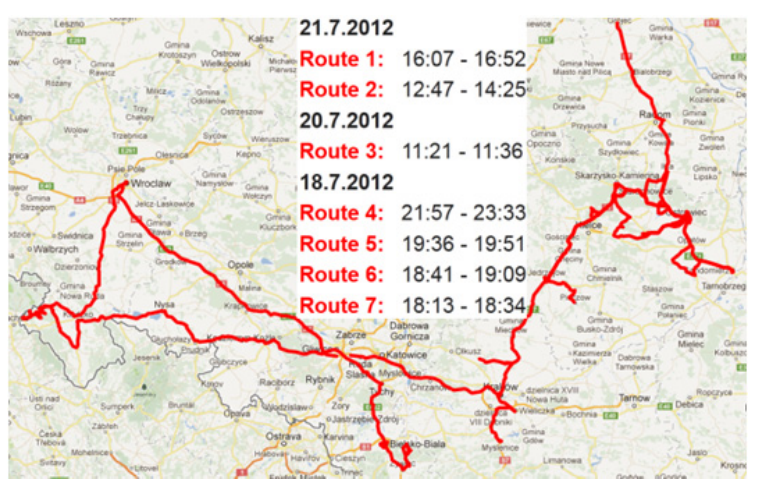

Figure 5: Displaying tracks on the map.

\section{RESULTS}

We measure the time spent between sending request to the system and presenting the result to the user. The time elapsed from user's query to the time of displaying the tracks on the screen using our system is compared with the same system that does not have reduction.

In all measurements, we ignore the time needed for data transfer. However, in weak internet access this might become bottleneck, and therefore, we design the system so that it minimizes data transfer. That allows using the system on computers with slower internet connection as well as on tablets that usually have limited bandwidth.

Table 1: Collections used for our experiments.

\begin{tabular}{|l|cccc|}
\hline User & Tracks & Points & $\begin{array}{c}\text { Length } \\
(\mathrm{km})\end{array}$ & $\begin{array}{c}\text { Duration } \\
(\mathrm{h})\end{array}$ \\
\hline Pasi & 784 & $1,216,039$ & 8,535 & 669 \\
Karol & 650 & $1,015,939$ & 9,655 & 442 \\
Radu & 429 & 613,684 & 4,604 & 188 \\
\hline
\end{tabular}

We present measurements for 3 sample users from Table 1. The original tracks consist of large number of points. In MOPSI, there are users having over one million points, which shows the need for reducing the number of points being displayed as none of the browser could handle such large number of points (Chen et al., 2009). In Table 2, we show the number 
of points from the original tracks within the selected time period and the number of points from the approximated tracks. The zoom level of the map is selected in such manner that all the tracks are visible on the map.

Figure 6 presents the time needed to display tracks in a selected period for three test users. The process is divided into three phases: querying database, computing bounding box and drawing in browser. Results show that the time needed for showing all the tracks of the user with the biggest collection is about 2.5 seconds.

Table 2: Number of points in original (left) and in the approximated tracks (right) in the selected time period for user Pasi.

\begin{tabular}{|l|cc|}
\hline & Original & Approximated \\
\hline all & $1,216,039$ & 9,064 \\
year & 424,709 & 3,088 \\
month & 46,669 & 331 \\
week & 11,204 & 903 \\
recent & 3,328 & 141 \\
\hline
\end{tabular}

Figure 7 shows average time percentages spent in each of the three phases. Querying data takes most of the time. Calculating bounding box is a fast process that additionally speeds up drawing tracks on map, so that it takes only $14 \%$ of time.

The approximation algorithm is necessary to reduce the number of points displayed. Without it, it is not possible to display all tracks because the web browser would crash. The number of points browsers can handle depends on available resources. Displaying thousands of points significantly slows down web browsers. Nevertheless, even if browser can display all the points in tracks, the time needed for the process increases.

Table 3: Size of files (in bytes) with original and approximated tracks for user Karol.

\begin{tabular}{|l|c|c|}
\hline & Original & Approximated \\
\hline week & 14.000 & 148 \\
\hline month & 346.000 & 2280 \\
\hline year & 4.056 .000 & 69.000 \\
\hline all & 11.595 .000 & 129.000 \\
\hline
\end{tabular}

Bigger number of points slows down the bounding box algorithm and often leads to memory issues. Moreover, approximation algorithm reduces files sizes as shown in Table 3 and preserves bandwidth used to retrieve data from server.
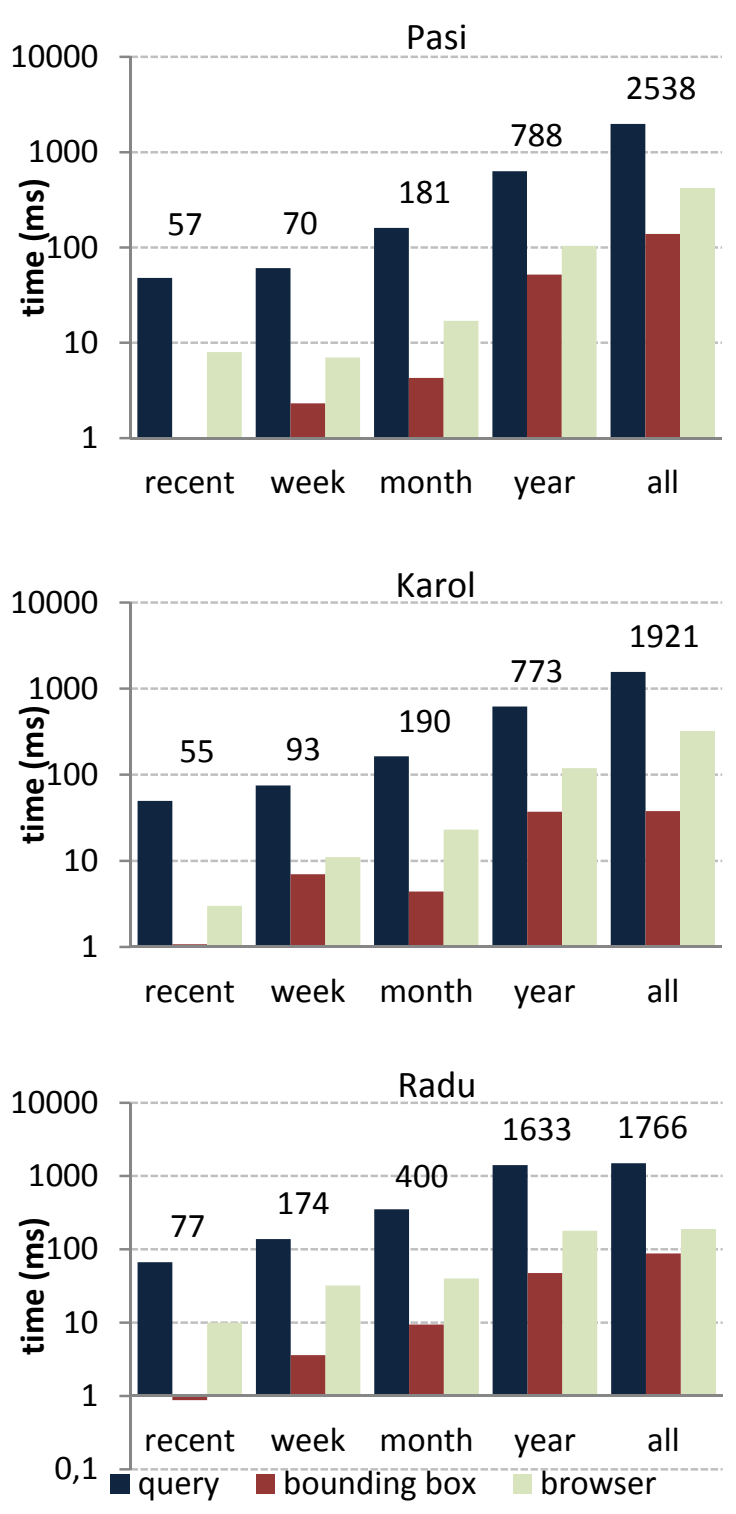

Figure 6: Display times of track collection for users Pasi, Karol and Radu.

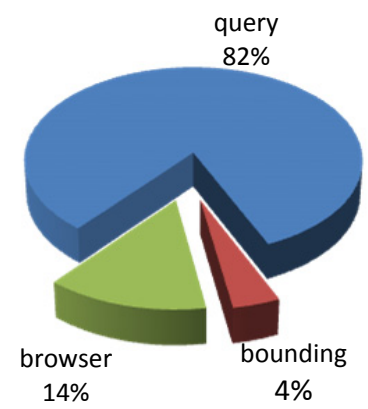

Figure 7: Average time percentage used for performing each operation of the system. 
Experiments show that applying bounding box decreases time needed to draw tracks on map. Fig. 8 shows a sample case from the experiments. In this case the same set of tracks was requested at the same zoom level, but the map was focused in two different places, Finland and Poland. In Finland the collection of tracks is big, whereas in Poland there are only several tracks available. Because of applying the bounding box solution, not all the tracks have to be displayed and the time to show the tracks when map shows fewer tracks (Poland area) is significantly shorter. Figure 8 also shows how reducing number of points affects the display time.
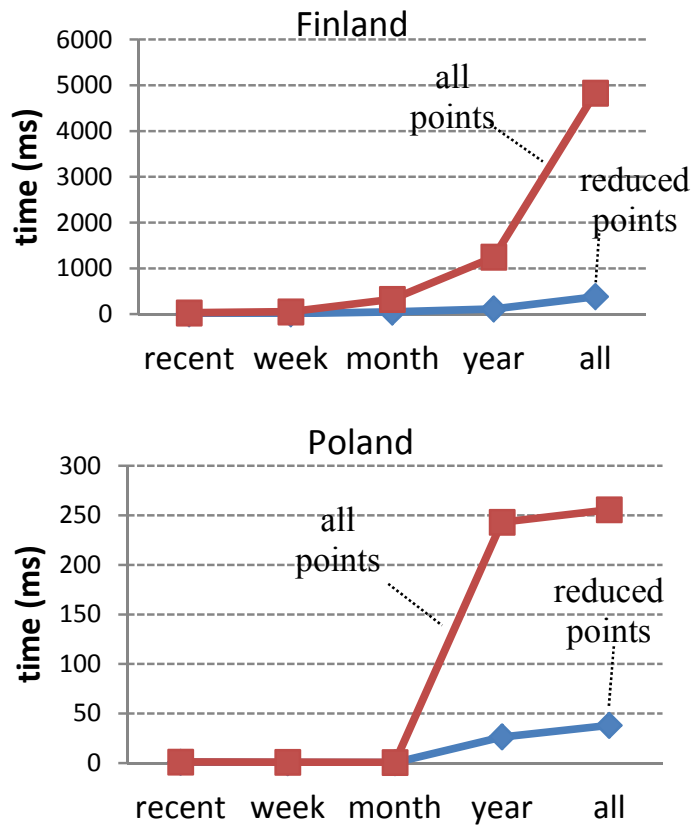

Figure 8: Example of querying the same track collection the same zoom level and focused in Finland (large collection, top) and Poland (small collection, bottom).

In comparison with the existing web based systems for visualizing GPS tracks, our system can display data consisting of significantly more points. For example, a track with about 10.000 points is displayed by our system in 1 second whereas GPS visualizer (www.gpsvisualizer.com) and GMapGis (www.gmapgis.com) need approximately 5 seconds. Moreover, user interaction is not slowed down in our system, when large number of points being is displayed.

\section{SUMMARY}

We presented a complete real time system to collect and visualize GPS tracks. Our motivation is to offer a system that is capable of handling large amount of GPS data so that user can access them in real time. The results show that our system is efficient even with large point collection. The most important part is the algorithm reducing the number of points to be displayed. Combined with a bounding box solution, the requested tracks can be accessed within about 2.5 seconds and the collection can be panned and zoomed with insignificant delay. The developed system can be used as a basis for more advanced analysis of GPS tracks, such as similarity and movement type detection.

Although, the system is efficient, there are still ways to improve it. For instance, now we reduce the number of points of one track only, but not when multiple tracks are overlapped. Further improvement could be achieved by clustering partial track segments. Moreover, the query phase should be optimized to minimize time needed to retrieve data.

\section{REFERENCES}

Alahakone, A. U., Ragavan, V. Geospatial Information System for Tracking and Navigation of Mobile Objects. ICAIM 09. Singapore, July, 2009.

Ananthanarayanan, G., Haridasan, M., Mohomed, I, Terry, D., Chandramohan, A. T. StarTrack: a Framework for Enabling Track-Based Application. ICMAS 09. Kraków, Poland, June 2009.

Chen, M., Xu, M., Fränti, P. A Fast O(N) Multi-resolution Polygonal Approximation Algorithm for GPS Trajectory Simplification. IEEE Trans. on Image Proc. 21(5). 2012.

Chen, Y., Jiang, K., Zheng, Y., Li, Ch., Yu, N. Trajectory Simplification Method for Location-based Social Networking Services. Int. Workshop on Location Based Social Network. Seattle, USA, November 2009.

Haridasan, M., Mohomed, I., Terry, D., Chandramohan, A. T., Li, Z. StarTrack Next Generation: A Scalable Infrastructure for Track-Based Applications, 2010.

Jakobs, K., Pils, C., Wallbaum, M. Using the Internet in Transport Logistics - The Example of a Track \& Trace System. Networking ICN, 194-203, 2001.

Martín S., Cristóbal E.S., Gil R., Díaz G., Oliva N., Castro M., Peire J. Finding the Way: Services for a MultiView and Multi-Platform Geographic Information System. WEBIST (2), pp.267-270, 2008.

McCullough, A., James, P., \& Barr, S. (2011). A Service Oriented Geoprocessing System for Real Time Road Traffic Monitoring. Transactions in GIS, 15(5), 651665, 2011

Morris, S., Morris, A., Barnard, K. Digital Trail Libraries. ACM/IEEE-CS Joint Conf. on Digital Libraries, pp. 63-71, June, 2004.

Waga, K., Tabarcea, A., Chen, M., Fränti, P. Detecting 
Movement Type by Route Segmentation and Classification. CollaborateCom, Pittsburgh, USA, October 2012.

Waga, K., Tabarcea, A., Mariescu-Istodor R., Fränti, P. System for Real Time Storage, Retrieval and Visualization of GPS Tracks. ICSTCC, Sinaia, Romania, October 2012.

Zheng, Y., Wang, L., Zhang, R., Xie, X., Ma, W.-Y. GeoLife: Managing and Understanding Your Past Life over Maps. Int. Conf. on Mobile Data Mgmt., Beijing, China, April 2008. 